\title{
Revisando a Síndrome de ALCAPA dos Tipos Infantil e Adulto: as Diferenças Estão nos Detalhes!
}

\author{
Ver artigos relacionados \\ nas páginas 438 e 446
}

\author{
Paolo Angelini ${ }^{1}$
}

0 dois artigos referentes à origem anômala da artéria coronária esquerda na artéria pulmonar (anomalous origin of the left coronary artery from the pulmonary artery - ALCAPA), publicados na atual edição da Revista Brasileira de Cardiologia Invasiva $(\mathbf{R B C I})^{1,2}$, trazem à tona a discussão sobre as contrastantes formas de apresentação, a fisiopatologia e os prognósticos dessas formas distintas de ALCAPA.

O primeiro caso ${ }^{1}$ ilustra uma apresentação típica do tipo infantil de ALCAPA, com grave disfunção do ventrículo esquerdo em paciente com 2 anos de idade, enquanto o segundo ${ }^{2}$ ilustra um caso clássico do tipo adulto, acompanhado por função preservada do ventrículo esquerdo, com angina de esforço manifestada tardiamente e que foi controlada com tratamento clínico.

Na verdade, os dois casos têm essencialmente a mesma anatomia: uma artéria coronária esquerda dominante, que anatomicamente se origina da artéria pulmonar, alimentada retrogradamente por uma rica rede de vasos colaterais fornecidos por uma grande artéria coronária direita. Os autores do primeiro caso ${ }^{1}$ relatam que a criança estava assintomática, com a função do ventrículo esquerdo criticamente deteriorada, e que, eventualmente, durante o primeiro mês da vida, sofrera eventos isquêmicos graves que não foram clinicamente reconhecidos, mas que mesmo assim foi capaz de sobreviver. A segunda paciente ${ }^{2}$, com 42 anos de idade, apresentava função ventricular normal quando começou a se queixar de angina aos esforços.

A seguir, são revisados, resumidamente, os aspectos básicos dessa afecção fascinante: a) embriogênese e variações anatômicas da ALCAPA, e b) fisiopatologia variável, levando a manifestações clínicas diferentes da ALCAPA.

\section{Embriogênese e variações anatômicas da ALCAPA}

A síndrome de ALCAPA constitui-se, essencialmente, da localização anômala do óstio da coronária esquerda na artéria pulmonar. Isso provavelmente é causado por uma alteração embrionária patológica da população de células da crista neural (CCN) cardíaca do embrião ainda em sua fase inicial ${ }^{3}$. Essas células migram da porção lateral do tubo neural embrionário em direção ao tronco aortopulmonar comum, contribuindo para a formação de arco aórtico, válvulas na aorta e na artéria pulmonar e suas camadas mediais vasculares, posição e origem das artérias coronárias e de seus óstios, além de fibras neurovegetativas do coração ${ }^{3}$.

Uma das possíveis conseqüências clínicas esperadas da falência das $\mathrm{CCN}$ é o desenvolvimento de valvas aórticas bicúspides, freqüentemente associadas a anomalias coronárias. Experimentalmente, Sans-Coma et al. ${ }^{4}$ demonstraram, em famílias de hamsters, que esses dois defeitos estão geneticamente associados. De forma coerente, o primeiro caso $^{1}$ apresentou tanto a síndrome de ALCAPA como uma valva aórtica bicúspide. Recentemente, um amplo espectro com variantes anatômicas da ALCAPA tem sido descrito: uma artéria coronária esquerda ectópica pode se originar tanto do seio de Valsalva posterior esquerdo (o modo mais freqüente, como apresentado nos dois casos mencionados $^{1,2}$ ) como do seio esquerdo em sua face não anterior ou, ainda, potencialmente, do seio coronariano anterior direito ${ }^{5}$, além do tronco principal da artéria pulmonar e seus ramos ${ }^{3}$.

Os parâmetros fundamentais a serem considerados na descrição de um caso de ALCAPA são: a) o território miocárdico dependente da artéria coronária esquerda (quanto maior, piores serão as conseqüênci-

\footnotetext{
1 St. Luke's Hospital, Texas Heart Institute, Baylor College of Medicine, Houston, Texas, Estados Unidos.

Correspondência: Paolo Angelini. 6624 Fannin, Ste 2780, Houston, TX 77030 - USA

E-mail: pangelini@leachmancardiology.com

Recebido em: 15/11/2007 • Aceito em: 20/11/2007
} 
as fisiológicas e clínicas); b) o grau de circulação colateral epicárdica (que desvia o sangue diretamente para a artéria pulmonar); e c) a presença de lesões obstrutivas na artéria coronária esquerda. Lesões ostiais do tronco da coronária esquerda são relativamente mais freqüentes em pacientes idosos com ALCAPA, o que pode influenciar favoravelmente a apresentação clínica. É possível que o segundo paciente ${ }^{2}$ apresentasse uma lesão do óstio coronariano esquerdo, o que foi sugerido pela observação das duas imagens publicadas no manuscrito $^{2}$. Obviamente, essa suposição deve ser ou não confirmada por provas diagnósticas específicas, tais como medições da velocidade de fluxo por meio de Doppler ou angiotomografia computadorizada das artérias coronárias. A obstrução da artéria coronária esquerda resultaria em melhora significativa na razão de fluxo entre as duas circulações paralelas (as resistências são baixas no fluxo da fístula para a artéria pulmonar e altas no fluxo epicárdico de nutrição do miocárdio), com vantagens para a perfusão miocárdica ${ }^{3}$.

\section{Fisiopatologia e manifestações clínicas}

Como já descrito na literatura ${ }^{3}$, as repercussões clínicas da ALCAPA só aparecem no período pósnatal, já que, durante a vida fetal, as pressões pulmonares e sistêmicas são iguais e o fluxo coronário é, assim, assegurado (anterógrado da artéria pulmonar), embora a saturação de oxigênio seja mais baixa que na aorta fetal. O período crítico ocorre no primeiro mês após o nascimento, quando os vasos pulmonares sofrem maturação rápida, e a circulação coronária da artéria pulmonar fica deficiente porque transporta sangue com baixas concentrações de oxigênio e, o mais importante, porque a pressão de perfusão pulmonar fica insuficiente. A partir desse momento, o ventrículo esquerdo se torna agudamente isquêmico e deficiente (determinado por processo isquêmico agudo ou subagudo e pelo desenvolvimento de discinesia apical ou difusa, insuficiência mitral, baixo débito cardíaco e choque, como descrito no primeiro caso $^{1}$ em uma forma de apresentação moderada). Esse estado foi claramente caracterizado como um exemplo de miocárdio hibernante, condição que é comum e substancialmente reversível para próximo da normalidade, até na vida adulta, após a correção cirúrgica ${ }^{3}$. O que é de fato crítico para ser considerado é que a pressão de perfusão, muito mais que a saturação de oxigênio, é fundamental na conservação da função ventricular. Por exemplo, em contraste com os quadros de ALCAPA, a transposição clássica dos grandes vasos se caracteriza por baixos índices de saturação de oxigênio nas artérias coronárias, mas pressão de perfusão coronária normal, que determina a preservação da função ventricular. No momento de agudização da crise infantil da ALCAPA, a mortalidade chega em seu pico, que, na ausência do reparo cirúrgico, atinge 50\% a $70 \%$, especialmente naqueles casos em que a artéria coronária esquerda é dominante. Superado esse mo- mento, a criança que sobrevive tende a se adaptar e seu estado tende a se estabilizar por dois mecanismos: a) reduzindo a demanda (o paciente em geral fica instintivamente sedentário, o que com freqüência mascara a condição em especial a olhos não instruídos, como no primeiro caso); e b) pelo desenvolvimento progressivo de uma rica circulação colateral, que se origina de uma artéria coronária direita normal, mantendo estado de hibernação crônica daquele território miocárdico dependente (caracterizado por funções sistólica e diastólica reduzidas, e também do metabo-

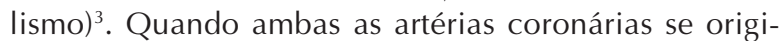
nam da pulmonar, as colaterais não podem se desenvolver e a sobrevivência é dependente da maturação vascular pulmonar. Somente os pacientes com hipertensão pulmonar (em decorrência de altas resistências vasculares, de falência ventricular esquerda, de regurgitação mitral ou de grande canal arterial patente [ducto de Botalli]) serão capazes de sobreviver ${ }^{3,5}$. O paciente raro com ALCAPA é aquele oligossintomático ou assintomático na vida adulta, sem disfunção ventricular significativa ou regurgitação mitral. Em geral, esse paciente se apresenta com estenose da artéria coronária esquerda (como discutido acima) ou com circulação colateral pouco usual, em que o fluxo de sangue da artéria coronária direita tende a passar pelo nível arteriolar coronário esquerdo antes de atingir o tronco da artéria coronária esquerda e drenar na pulmonar. Em tais casos, a circulação colateral é menos evidente na angiografia. Em tais situações, mesmo exames provocativos de isquemia, como a cintilografia miocárdica, podem ser negativos. Esses são os casos (bastante excepcionais) que têm evolução favorável quando tratados clinicamente. Na minha experiência pessoal, a cateterização seletiva do tronco da artéria coronária esquerda pela artéria pulmonar (com cateteres de angioplastia) permite medir a saturação do oxigênio do sangue ali drenado. Somente em pacientes com prognóstico favorável essa saturação de oxigênio é mais baixa que a sistêmica (dados não publicados). Os pacientes assintomáticos (tipicamente aqueles que levam vida sedentária), mas que apresentam disfunção ventricular grave ou grande área de isquemia, não devem ser tratados clinicamente. De forma irreversível (mesmo após a correção cirúrgica tardia), eventualmente ainda podem ocorrer dano miocárdico e morte súbita. Provas funcionais, como cintilografia miocárdica e monitorização com Holter, devem ser costumeiramente executadas, mesmo na ausência de sintomas, e com periodicidade anual em qualquer adulto portador de ALCAPA, a quem inicialmente deve ser indicado tratamento medicamentoso.

\section{CONCLUSÃO}

A síndrome de ALCAPA representa grande desafio tanto para o coração da criança como para o médico. A principal atitude clínica é reconhecer a presença de isquemia em uma criança (tarefa clínica, de fato, difí- 

2007; 15(4): 334-336.

cil) e, então, conhecer os detalhes anatômicos e funcionais, bastante variáveis nos diferentes pacientes e criticamente importantes na determinação do espectro de manifestações clínicas, e o planejamento das soluções cirúrgicas.

\section{REFERÊNCIAS BIBLIOGRÁFICAS}

1. Bregagnollo IF, Oliveira GS, Bonatto RC, Carvalho FC. Origem anômala de artéria coronária esquerda do tronco pulmonar: síndrome de Bland-White-Garland. Relato de caso. Rev Bras Cardiol Invas. 2007;15(4):438-42.
2. Giusti I, Yordi LM, Sarmento-Leite R. Síndrome da BlandWhite-Garland. Rev Bras Cardiol Invas. 2007;15(4):446.

3. Angelini P. Coronary artery anomalies: a comprehensive approach. New York:Lippincott, Williams \& Wilkins;1999. p.11-190.

4. Sans-Coma V, Arqué JM, Durán AC, Cardo M. Origin of the left main coronary artery from the pulmonary trunk in the Syrian hamster. Am J Cardiol. 1988;62(1):159-61.

5. Angelini $P$. The miraculous survival of an exceptional baby: can a heart manage to function with anomalous origin of both coronary arteries from the pulmonary artery? Tex Heart Inst J. 2005;32(3):351-3. 